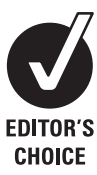

${ }^{1}$ Department of Emergency Medicine, University of Cincinnati College of Medicine, Cincinnati, Ohio, USA;

${ }^{2}$ Department of Emergency Medicine, Allegheny General Hospital, Pittsburgh, Pennsylvania, USA

Correspondence to:

Dr A Venkat, Department of

Emergency Medicine, Allegheny General Hospital, 320 East North Avenue, Pittsburgh, PA 15212 USA; avenkat@wpahs.org

Preliminary data analysis was presented at the Society of Academic Emergency Medicine Annual Meeting, Chicago, Illinois, 17 May 2007.

Accepted 3 March 2008

\title{
Derivation of a risk assessment tool for emergency department patients with sickle cell disease
}

\author{
A W Bernard, ${ }^{1}$ C J Lindsell, ${ }^{1}$ A Venkat ${ }^{2}$
}

\begin{abstract}
Introduction: Sickle cell patients commonly present to the emergency department (ED). Identifying those requiring admission and those who can safely be discharged is difficult. It was hypothesised that ED variables predictive of $96-\mathrm{h}$ adverse sickle cell patient outcomes are identifiable.
\end{abstract}

Methods: This observational cohort study included all adult sickle cell patient visits (1 June 2004-31 May 2005) to two ED. Patients were identified by ICD-9 codes of vaso-occlusive crisis and lists from treating haematologists. ED charts were abstracted for history, physical examination, laboratory/imaging data and outcomes. Outcomes were hospitalisation within $96 \mathrm{~h}$ of ED presentation for transfusion/antibiotic treatment, acute chest syndrome, or aplastic or sequestration crisis. Logistic regression was used to derive a risk score, which was tested in a validation cohort. The area under the receiver operating curve (AUC) was used to measure score performance.

Results: There were 884 ED visits by 125 patients (mean age 36 years $/ 55 \%$ female $/ 58 \%$ homozygous sickle cell disease). 199 ED visits had one or more outcome (197 transfusion/antibiotic treatment, 71 acute chest syndrome, and one aplastic crisis). The risk score included sickle variant, chest pain, chills, pain dissimilar to past, temperature $\left(<36^{\circ} \mathrm{C} />38^{\circ} \mathrm{C}\right)$, oxygen saturation $(<95 \%)$, haemoglobin $(<10 \mathrm{~g} / \mathrm{dl})$, urine nitrites and chest $x$ ray abnormality. The score had an AUC of $0.816(95 \% \mathrm{Cl}$ 0.778 to 0.854$)$ in the derivation cohort, $0.824(95 \% \mathrm{Cl}$ 0.760 to 0.889 ) in the validation cohort.

Conclusion: Those ED variables predictive of 96-h adverse sickle cell patient outcomes can be identified and combined into a risk score. Prospective validation is necessary before any clinical decision-making based on this score.

Sickle cell patients commonly present to the emergency department (ED). ${ }^{12}$ It is currently unclear what aspects of the ED evaluation contribute to accurate risk stratification. ${ }^{3-7}$ Life-threatening sickle cell complications (acute chest syndrome, splenic sequestration, or aplastic crisis) are difficult to recognise on initial ED evaluation. Acute chest syndrome is diagnosed after admission for sickle cell pain crisis approximately $50 \%$ of the time; the average time from admission to diagnosis in these patients is 2.5 days. $^{89}$

We hypothesise that, from among variables routinely collected in the ED evaluation of the sickle cell patient, a limited number relate to the need for acute medical intervention following an $\mathrm{ED}$ visit and that combining the variables identified into a risk model, it is possible to identify those sickle cell patients who will require acute medical care within $96 \mathrm{~h}$ of their ED visit. We hypothesised that those variables predictive of requiring acute medical intervention would be derived from history, physical examination and vital signs, with routine laboratory and radiographic data adding little to the predictive model.

\section{METHODS \\ Study design}

This was a retrospective, observational study of all adult sickle cell patient ED presentations from 1 June 2004 to 31 May 2005 to an urban, academic centre (85000 visits) and an urban community centre (42 000 visits). Subjects were identified from patient lists provided by treating haematologists. ED billing records were also searched for International Classification of Disease, version 9 codes (282.42, 282.6, 282.62, 282.64 and 282.69) corresponding to sickle cell or vaso-occlusive crisis. From this patient list, electronic medical records were searched for all ED visits during the study time frame. Patients were excluded if they were under 18 years of age. The study was approved by the local Institutional Review Boards of both settings.

\section{Data collection and processing}

Data elements, including demographic information, theoretically important clinical variables based on previous research ${ }^{3-9}$ and outcome measures, were abstracted to a standardised case report form. Data were abstracted by one of two emergency physicians using explicit definitions for each variable. Missing data elements were recorded as such.

\section{Outcome measures}

The primary outcome measures were hospitalisation with acute chest syndrome, aplastic crisis, splenic sequestration and blood transfusion or antibiotic administration within $96 \mathrm{~h}$ of ED presentation. Outcomes were captured by review of discharge diagnosis and hospital records for the current visit and by review of medical records for any repeat visits occurring within $96 \mathrm{~h}$. The 96-h time frame was chosen to capture occult acute chest syndrome related to the initial encounter and also represents a realistic time frame for outpatient follow-up. Although antibiotic administration and blood transfusion are subject to physician discretion, they were chosen because they identify ill patients. At the study institutions, similar to most other hospitals, the use of these therapies is without precise protocols because of inherent difficulties of creating guidelines for such treatment. Infection is considered an important trigger 
Table 1 Demographics and sickle variant for the derivation and validation datasets

\begin{tabular}{lllll}
\hline & $\begin{array}{l}\text { Derivation set } \\
\mathbf{n}=\mathbf{9 4} \text { (670 visits) }\end{array}$ & Range or percentage & $\begin{array}{l}\text { Validation set } \\
\mathbf{n}=\mathbf{3 1} \text { (214 visits) }\end{array}$ & Range or percentage \\
\hline Median age, years & 32.5 & $(19-66)$ & 39 & $(22-56)$ \\
Male & 43 & $(45.7)$ & 13 & $(41.9)$ \\
Female & 51 & $(54.3)$ & 18 & $(58.1)$ \\
SS & 54 & $(57.4)$ & 18 & $(58.1)$ \\
SC & 21 & $(22.3)$ & 7 & $(22.6)$ \\
S Thal & 10 & $(10.6)$ & 2 & $(6.5)$ \\
S Los Angeles & 0 & $(0.0)$ & 1 & $(3.2)$ \\
Trait & 0 & $(0.0)$ & 1 & $(3.2)$ \\
Unknown & 9 & $(9.6)$ & 2 & $(6.5)$ \\
Median no of visits & 6 & $(1-67)$ & 8 & $(1-60)$ \\
\hline
\end{tabular}

Data are medians and ranges or frequencies and percentages.

SC, sickle cell haemogoblin C disease; S Los Angeles, sickle Los Angeles disease; SS, homozygous sickle cell disease; S Thal, sickle cell beta thalassaemia disease; Trait, heterozygous sickle cell disease.

of vaso-occlusive crisis and not all infection that requires antibiotics can be identified by objective testing. The limitation of blood cultures in pneumonia is well established and bronchoscopy is not a practical part of routine clinical practice. ${ }^{10}$ Other infectious processes, such as bacterial sinusitis and bronchitis, also lack clinically practical gold standards. Similarly, the use of blood transfusion by a haematologist is guided by a constellation of clinical and laboratory findings. It is therapeutic in a variety of complications of sickle cell disease and can signify an ill patient. ${ }^{11}$ However subjective the use of these therapies might be, identification of patients a haematologist deems ill enough to require treatment is important to emergency physicians.
Acute chest syndrome was defined as a new pulmonary infiltrate on chest radiograph in conjunction with at least one clinical sign of pulmonary disease (fever, tachypnoea, chest pain, shortness of breath, cough). ${ }^{89}$ At these institutions, patients meeting these criteria are sometimes diagnosed with pneumonia. Therefore, hospital discharge diagnosis was insufficient for the study outcome.

\section{Primary data analysis}

The sample was randomly partitioned into a derivation and validation set at the patient level, ie, a patient could only appear in the derivation or validation set, but not both. All ED visits for $75 \%$ of patients were used in the derivation set, whereas all ED

Table 2 Best fit multivariable model based on history and physical and the best fit model incorporating laboratory findings

\begin{tabular}{|c|c|c|}
\hline Predictor & Reference group & Odds ratios $(95 \% \mathrm{Cl})$ \\
\hline SC & S Thal & 2.66 (1.12 to 6.31$)$ \\
\hline SS & & 2.31 (1.08 to 4.95$)$ \\
\hline Other/unknown & & 9.17 (3.56 to 23.65$)$ \\
\hline Pain in chest & No pain in chest & 2.35 (1.55 to 3.57$)$ \\
\hline Pain similar to previous & Pain not similar to previous & $0.60(0.40$ to 0.91$)$ \\
\hline Chills & No chills & 3.85 (1.69 to 8.79$)$ \\
\hline Temperature abnormal & Temperature normal & $4.37(2.06$ to 9.25$)$ \\
\hline Temperature not done & & $0.74(0.25$ to 2.21$)$ \\
\hline Pulse oximetry abnormal & Pulse oximetry normal & $4.72(2.63$ to 8.50$)$ \\
\hline Pulse oximetry not done & & $1.62(0.87$ to 2.99$)$ \\
\hline SC & S Thal & 2.97 (1.15 to 7.65$)$ \\
\hline SS & & $1.95(0.83$ to 4.56$)$ \\
\hline Other/unknown & & 8.09 (2.84 to 23.08 ) \\
\hline Pain in chest & No pain in chest & 1.83 (1.13 to 2.97$)$ \\
\hline Pain similar to previous & Pain not similar to previous & $0.54(0.34$ to 0.85$)$ \\
\hline Chills & No chills & $2.42(0.95$ to 6.13$)$ \\
\hline Temperature abnormal & Temperature normal & 5.35 (2.29 to 12.49$)$ \\
\hline Temperature not done & & $0.80(0.23$ to 2.70$)$ \\
\hline Pulse oximetry abnormal & Pulse oximetry normal & $3.56(1.85$ to 6.85$)$ \\
\hline Pulse oximetry not done & & 1.87 (0.93 to 3.74$)$ \\
\hline Haemoglobin $<10$ & Haemoglobin $\geqslant 10$ & 2.88 (1.68 to 4.94$)$ \\
\hline Haemoglobin not measured & & 0.36 (0.09 to 1.38$)$ \\
\hline Nitrite positive & Nitrite negative & $4.11(1.35$ to 12.56$)$ \\
\hline Nitrite not assessed & & $0.80(0.49$ to 1.31$)$ \\
\hline Chronic abnormality & Normal on CXR & $1.82(1.01$ to 3.27$)$ \\
\hline Acute abnormality & & 5.75 (2.69 to 12.31$)$ \\
\hline Not done & & 0.71 (0.35 to 1.42$)$ \\
\hline
\end{tabular}

CXR, chest $x$ ray; SC, sickle cell haemogoblin C disease; SS, homozygous sickle cell disease; S Thal, sickle cell beta thalassaemia disease. 


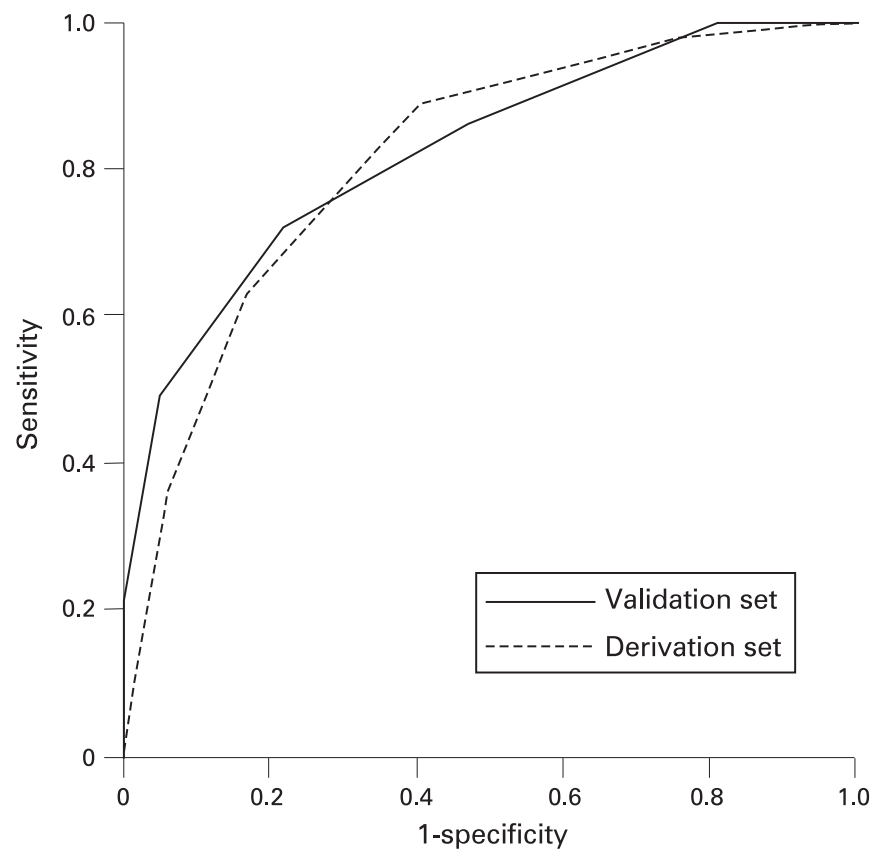

Figure 1 Receiver operating characteristic curves showing the performance of the score in the derivation and validation datasets.

visits for the remaining $25 \%$ of patients were used in the validation set. Patient demographic characteristics at the first visit have been used to describe the sample. It was assumed that clinical factors change between visits depending on patient condition, and that disposition and treatment decisions change at each visit depending on the physicians. Therefore, further analysis considered visits independent.

With the derivation set, univariable logistic regression was used to determine which history, physical and vital sign measures were associated with increased odds of an outcome. Next, a multivariable model was constructed based only on independently predictive variables. To maximise the interpretability of the multivariable model and avoid bidirectional effects, vital signs were categorised as normal or abnormal. Vital signs were defined as abnormal if they exceeded the following limits: temperature less than 36 or more than $38^{\circ} \mathrm{C}$, pulse less than 60 or more than 100 beats per minute, respiratory rate less than 12 or more than 20 breaths per minute, systolic blood pressure less than 90 or more than $140 \mathrm{~mm} \mathrm{Hg}$, diastolic blood pressure less than 60 to more than $90 \mathrm{~mm} \mathrm{Hg}$, and pulse oximetry less than 95\% oxygen saturation. Models were constructed and compared using nonautomated stepwise selection of variables with statistical significance combined with effect size used to determine variable addition or removal.

As a result of the use of retrospective chart review methodology, data were expected to be missing, not always randomly. For signs and symptoms, a lack of documentation was presumed as indicating a lack of presence of that sign or symptom. For test results that were classified as normal or abnormal, "missing" was treated as a category for comparisons to avoid the exclusion of a significant proportion of cases. Only eight variables evaluated were continuous in nature; for these variables, cases were excluded for univariable analyses. If found to be a predictor of outcomes, they were categorised as normal, abnormal and test not done, as appropriate.
For laboratory and radiological data, each finding was added independently to the best fit model based on history, physical examination and vital signs. Variables found to add significantly to the model containing elements only from the history, physical examination and vital signs were considered for inclusion in the expanded model. The expanded model was constructed in a similar manner to the basic model, with the exception that all variables in the basic model were forced into the final model. Once the model was finalised, it was tested using the validation set.

Receiver operating characteristic (ROC) curves were generated to assess model accuracy. Diagnostic test statistics were computed for dichotomous decision points; 95\% CI of these statistics were computed using the score method.

\section{RESULTS}

\section{Study subject characteristics}

There were 884 patient visits for 125 patients during the study period (median three visits per patient). The mean age was 36.3 years; 56 (44.8\%) were men. Sickle variant was homozygous sickle cell disease (SS) in 72 (57.6\%), sickle cell haemogoblin C disease (SC) in 28 (22.4\%), sickle cell beta thalassaemia disease (S Thal) in 12 (9.6\%), sickle Los Angeles disease in one $(0.8 \%)$, heterozygous sickle cell disease (trait) in one $(0.8 \%)$ and unknown in 11 (8.8\%) (table 1$)$.

Of 284 admitted visits, 199 were found to have one or more of the outcomes. There were 197 admissions for transfusion or antibiotic administration, 71 for acute chest syndrome and one for aplastic crisis; there were no splenic sequestration events. Of these events, $33(16.6 \%)$ were in patients returning to the ED after an initial visit that did not result in admission to the hospital. The majority of ED visits were for sickle cell pain crisis.

\section{Main results}

The best fit multivariable model from history and physical examination included sickle variant, chest pain, pain not similar to previous, abnormal temperature and abnormal pulse oximetry as predictors of outcomes (table 2). The C-statistic for this multivariable model was 0.764 (95\% CI 0.720 to 0.804).

When adding laboratory and imaging parameters to this model, haemoglobin, urine nitrites and chronic and acute chest $x$ ray abnormalities were associated with increased odds of adverse outcome. Haemoglobin less than $10 \mathrm{~g} / \mathrm{dl}$ was considered to be clinically relevant in this population. Simple transfusion for the sole indication of anaemia would be unusual at a level more than $10 \mathrm{~g} / \mathrm{dl}$ due to the risk of hyperviscosity and stroke. ${ }^{11}$ The C-statistic for this multivariable model (table 2) was 0.854 (95\% CI 0.823 to 0.886 ).

A scoring system, developed on the basis of the multivariable logistic regression model, was computed as the sum of the number of factors present. The test characteristics of the score at thresholds of more than two and more than four are shown (table 3). The area under the ROC curve for this score in the derivation set was $0.816(95 \%$ CI 0.778 to 0.854$)$. In the validation set, the area under the ROC curve was 0.824 (95\% CI 0.760 to 0.889 ) (fig 1 ).

\section{DISCUSSION}

The data in this study support the hypothesis that identifiable variables in the ED evaluation of adult sickle cell patients can be constructed into a risk assessment tool that has reasonable accuracy in an independent cohort. However, history, physical, and vital sign variables alone did not provide adequate accuracy, 
Table 3 Scoring system and diagnostic test characteristics of the score and physician judgement in the validation set, $95 \% \mathrm{Cl}$ are shown

\begin{tabular}{|c|c|c|c|}
\hline \multicolumn{3}{|l|}{ Risk factor } & Score if present \\
\hline \multicolumn{3}{|c|}{ SS, SC, other or unknown variant } & +1 \\
\hline \multicolumn{3}{|c|}{ Chest pain } & +1 \\
\hline \multicolumn{3}{|l|}{ Chills } & +1 \\
\hline \multicolumn{3}{|c|}{ Pain not similar to previous episodes } & +1 \\
\hline \multicolumn{3}{|c|}{ Temperature $<36^{\circ} \mathrm{C}$ or $>38^{\circ} \mathrm{C}$} & +1 \\
\hline \multicolumn{3}{|c|}{ Oxygen saturation $<95 \%$} & +1 \\
\hline \multicolumn{3}{|c|}{ Haemoglobin $<10$} & +1 \\
\hline \multicolumn{3}{|c|}{ Nitrites present on urinalysis } & +1 \\
\hline \multicolumn{3}{|c|}{ Abnormalities observed on chest $x$ ray } & +1 \\
\hline \multicolumn{3}{|c|}{ Total possible score } & 9 \\
\hline & Score $>2$ & Score $>4$ & Physician judgement \\
\hline Overall accuracy & 61.7 (54.8 to 68.2 ) & 82.7 (76.8 to 87.4 ) & 81.8 (75.8 to 86.6$)$ \\
\hline Sensitivity & 86.0 (73.7 to 93.3$)$ & 49.1 (35.8 to 62.6$)$ & 77.2 (63.8 to 86.8 ) \\
\hline Specificity & 52.9 (44.8 to 60.8$)$ & $94.9(89.9$ to 97.6$)$ & 83.4 (76.5 to 88.7$)$ \\
\hline False negative rate & $14.0(6.7$ to 26.3$)$ & 50.9 (37.4 to 64.2$)$ & 22.8 (13.2 to 36.2$)$ \\
\hline False positive rate & 47.1 (39.2 to 55.2$)$ & $5.1(2.4$ to 10.1$)$ & $16.6(11.3$ to 23.5$)$ \\
\hline Positive predictive value & 39.8 (31.2 to 49.1$)$ & $77.8(60.4$ to 89.3$)$ & $62.9(50.4$ to 73.9$)$ \\
\hline Negative predictive value & 91.2 (82.9 to 95.9$)$ & 83.7 (77.3 to 88.6$)$ & 91.0 (84.8 to 94.9$)$ \\
\hline
\end{tabular}

SC, sickle cell haemogoblin C disease; SS, homozygous sickle cell disease.

rejecting the secondary hypothesis. There are, however, several limitations.

Missing data elements from history and physical examination variables are presumed to be negative findings, but the possibility of the poor documentation of positive findings exists. This may have resulted in an underestimation of risk and thus decreased sensitivity of the model for some patients. Not all patients received every diagnostic test, which also affects the analysis; the absence of testing was associated with decreased odds of an outcome suggesting that missing testing was associated with decreased physician suspicion of a sickle cell crisis and resultant adverse outcome. The work-up bias present in our model is thus likely to be physician dependent.

Although data analysis was conducted using a prespecified strategy, the potential for bias exists because only one physician reviewed each patient encounter. The abstraction of adverse outcomes was limited to events occurring within the twohospital system. However, the majority of regional sickle patients primarily frequent the two systems included in this study. A disconnect between standard research terminology and clinical terminology in the diagnosis of acute chest syndromes used at the study sites may also have limited outcome evaluation. To overcome this, a precise definition of acute chest syndrome was established before abstraction and the criteria necessary to meet this definition were reviewed with every encounter.

Previous research in the adult patient population also had few aplastic or sequestration crises. It has been suggested that this was due to the exclusion of high-risk patients from those studies, ${ }^{5}$ but now appears to be more a reflection of the epidemiology of these outcomes as no patients were excluded in our study. The outcomes of blood transfusion and antibiotic administration have limitations in variability of use. ${ }^{11}$ These therapies were not linked to specific testing but are markers of ill patients and of those patients a risk score would want to capture.

The derivation of a simple sum score is a limitation of this study when a more complex weighted sum score is feasible. A weighted score based on the coefficients in the logistic model did not improve performance in the validation set (data not shown), and the simple score has more clinical utility due to its simplified nature. ${ }^{12}$

Despite these limitations, our findings help to identify sickle cell patients at risk of complications. Historically, the identification of life-threatening complications of sickle cell disease during an ED evaluation has not always been possible, highlighted by the fact that almost $50 \%$ of cases of acute chest syndrome are diagnosed only after admission to the hospital for presumed uncomplicated sickle cell pain crisis. ${ }^{8}$ These cases probably represent patients who presented in a prodromal period before the development of the acute chest syndrome. ${ }^{8}{ }^{13}$ For these reasons, the goal of the ED evaluation must not only be to identify those patients who present with life-threatening complications of sickle cell disease, but also those at immediate risk of developing complications.

Dogma suggests that aspects of that initial history and physical examination alone may aid in risk stratification, but there is little evidence to support such claims. ${ }^{14}$ Our research confirms that variables from the vital signs, history and physical examination were associated with adverse outcomes (tables 2 and 3). We found sickle cell variants SS, SC, and those recorded as other or unknown to be associated with adverse events when compared with S Thal. Patients with SS disease are known to have more severe disease and shorter life expectancy. ${ }^{15-18}$ Their association with adverse events is therefore expected. Patients with SC disease generally have milder disease and the association we found with adverse events may not be anticipated. ${ }^{16}$ Further study is necessary to determine the reasons for this association. Patients with unknown sickle cell variants or other variants also had greater odds of adverse events. One explanation is that a proportion of homozygous patients were not properly identified.

One survey of academic ED found high variability in the use of diagnostic testing, and diagnostic testing has been shown to be limited when ordered as a matter of routine..$^{2-7}$ Our analysis was designed to determine if a risk score could be created based upon vital signs, history and physical examination alone, and then to determine whether further diagnostic testing was useful or necessary. The test characteristics of the initial model were inadequate to forgo diagnostic testing. The C-statistic for our 
multivariable models improved from 0.764 (95\% CI 0.720 to $0.804)$ to 0.854 (95\% CI 0.823 to 0.886 ) with the addition of diagnostic testing. In particular, haemoglobin less than $10 \mathrm{~g} / \mathrm{dl}$, nitrites present on urinalysis and abnormalities observed on $x$ ray improved the test characteristics of our model (table 2).

This discrepancy from previous studies may be explained by methodology. Lopez et al ${ }^{3}$ found no difference in haemoglobin levels between admitted and discharged patients, and thus suggested that this testing was not useful. Admission may not account for, and is not specific to, life-threatening complications. Our study, with a larger sample size and focused on complication-oriented outcomes, found this test useful as part of a risk stratification score.

Previous reports that have conflicting results on the utility of chest radiography are limited by sample size and exclusion criteria. ${ }^{67}$ Our work, consistent with Pollack et al, ${ }^{6}$ suggests that chest radiographs detect illness not suspected on history and physical examination. Even in the absence of an acute radiographic abnormality, the chest radiograph has utility in a diagnostic evaluation. Chronic changes were found to be associated with the risk of adverse events. This may be a reflection of chronic lung disease as a predisposition to illness.

This investigation did show consistency with some earlier research. The reticulocyte count does not appear to be a useful test in adult sickle cell patients presenting to the ED. ${ }^{3-5}$ The utility of urinalysis in these patients was again confirmed. ${ }^{67}$ In our work, although testing was not linked to culture results, we found that a positive nitrite dipstick increased the risk of adverse outcomes. Although many of these nitrite-positive urinalyses will have a positive culture, some will not. ${ }^{19}$ The data suggest that even though some individual patients may in fact go on to be culture negative, the nitrite-positive group as a whole has an increased risk of adverse outcomes, probably due to the fact that many patients will be culture positive.

Prospective validation is necessary before any clinical decision-making based on this score. If this score is prospectively validated, however, diagnostic testing in patients with sickle cell disease may become more consistent. Haematological testing may increase for those physicians who do not routinely obtain blood work. Urinalysis, which is ordered on a routine basis by approximately $20 \%$ of emergency physicians, ${ }^{2}$ and chest radiography could potentially also increase in use. Future work should consider the decreased need to expose patients to radiation during $x$ ray when the score is either high or low based on other variables. It is also possible that admissions could be more specific and discharges safer with the use of this score. The score may also facilitate initiating simple therapies, such as incentive spirometry, which has been proved to prevent the development of the acute chest syndrome. ${ }^{20}$

\section{CONCLUSION}

This research demonstrates that variables predictive of adverse outcomes within $96 \mathrm{~h}$ of a sickle cell patient presenting to the ED can be identified. A simple risk score derived from these variables demonstrates reasonable accuracy in an independent sample. Prospective validation is necessary before any clinical decision-making related to this score.

Competing interests: None.

Ethics approval: The study was approved by the local Institutional Review Board of both settings.

AV was a member of the Department of Emergency Medicine at the University of Cincinnati at the time of performance of this study.

\section{REFERENCES}

1. Shankar SM, Arbogast PG, Mitchel E, et al. Medical care utilization and mortality in sickle cell disease: a population based study. Am J Hematol 2005;80:262-70.

2. Silbergleit R, Jancis MO, McNamara RM. Management of sickle cell pain crisis in the emergency department at teaching hospitals. J Emerg Med 1999;17:625-30.

3. Lopez BL, Griswold SK, Navek A, et al. The complete blood count and reticulocyte count-are they necessary in the evaluation of acute vasoocclusive sickle-cell crisis. Acad Emerg Med 1996;3:751-7.

4. Wells BL, Vizoli TL, Counselman FL. The reticulocyte count: is it needed for evaluating typical sickle cell crisis presenting to the ED? Am J Emerg Med 2002;20:69-70

5. Bernard AW, Venkat A. Lyons MS. Full blood count and reticulocyte count in painful sickle crisis. Emerg Med J 2006;23:302-3.

6. Pollack CV, Jorden RC, Kolb JC. Usefulness of empiric chest radiography and urinalysis testing in adults with acute sickle cell pain crisis. Ann Emerg Med 1991;20:1210-14.

7. Ander DS, Vallee PA. Diagnostic evaluation for infectious etiology of sickle cell pain crisis. Am J Emerg Med 1997;15:290-2.

8. Vichinsky EP, Neumayr, LD, Earles AN, et al. Causes and outcomes of the acute chest syndrome in sickle cell disease. N Engl J Med 2000;342:1855-65.

9. Bernard AW, Yasin Z, Venkat A. Acute chest syndrome of sickle cell disease. Hosp Physician 2007; 43:15-23, 44.

10. Kennedy M, Bates DW, Wright SB, et al. Do emergency department blood cultures change practice in patients with pneumonia? Ann Emerg Med 2005;46:393-400.

11. Wayne AS, Kevy SV, Nathan DG. Transfusion management of sickle cell disease. Blood 1993:81:1109-23.

12. Brehaut J, Graham I, Eagles D, et al. The acceptability of clinical decision rules: validation of the Ottawa Acceptability of Decision Rules Scale (OADRS). Acad Emerg Med 2007;14(5 Suppl 1):S49.

13. Vichinsky EP, Styles LA, Colangelo LH, et al. Acute chest syndrome in sickle cell disease: clinical presentation and course. Blood 1997;89:1787-92.

14. Freeman L. Sickle cell disease and other hemoglobinopathies: approach to emergency diagnosis and treatment. Emerg Med Pract 2001;3(12).

15. Platt OS, Brambilla DJ, Rosse WF, et al. Mortality in sickle cell disease-life expectancy and risk factors for early death. N Engl J Med 1994;330:1639-44.

16. Powars D, Chan LS, Schroeder WA. The variable expression of sickle cell disease is genetically determined. Semin Hematol 1990;27:360-76.

17. Platt OS, Thorington BD, Brambilla DJ, et al. Pain in sickle cell disease. Rates and risk factors. N Engl J Med 1991;325:11-16.

18. Castro 0, Brambilla DJ, Thorington B, et al. The acute chest syndrome in sickle cell disease: incidence and risk factors. Blood 1994;84:643-9.

19. Lammers RL, Gibson S, Kovacs D, et al. Comparison of test characteristics of urine dipstick and urinalysis at various cutoff points. Ann Emerg Med 2001;38:505-12

20. Bellet PS, Kalinyak KA, Shukla R, et al. Incentive spirometry to prevent acute pulmonary complications in sickle cell diseases. N Engl J Med 1995;333:699-703. 